\title{
Misplacement of the Nasogastric Tube into the Anterior Neck Potential Space
}

Marni Mahfuzah Abdul Wahid ${ }^{1}$, Nadiah Hamdan ${ }^{1}$, Nor Azura Ahmad ${ }^{1}$, Ameer Mukmeen Sezali ${ }^{1}$, Nur Fariza Ramly ${ }^{1}$, Abdul Hadi Mohamed ${ }^{1}$

${ }^{1}$ Department of Anaesthesiology and Intensive Care, Kulliyyah of Medicine, International Islamic University of Malaysia

Presenter: Marni Mahfuzah Abdul Wahid

Nasogastric tube insertion is a very common procedure performed in intensive care settings which require a basic skill. In mechanically ventilated patients, the blind technique may lead to inadvertent placement of the tube into the lung even when the endotrachial cuff was inflated. The preferred technique is using a laryngoscopy for direct vision during the insertion. The gold standard of NGT placement confirmation in mechanically ventilated patient is with chest radiograph. Other clinical confirmation tests are not as reliable in intubated patients. A 44 year old lady came in with huge anaplastic carcinoma of the thyroid with compression symptoms. Otherwise, she had an unremarkable other medical problem. She underwent palliative debulking surgery to remove part of the tumor. The tumor was very aggressive, few days postoperatively it caused tracheal erosion with subcutaneous emphysema around the neck and upper chest. Urgent tracheostomy was done. Following this operation, NGT was reinserted. The nasogastric insertion was uneventful and the placement of the tube was confirmed by aspiration of gastric content and syringe test. Enteral feeding was resumed afterward. A few hours later the patient complained of neck pain and tense swelling developed on her neck and upper chest. Whitish secretion was noted to seep out around the tracheostomy wound. Enteral feeding was withheld and chest radiograph showed coiled NGT in the potential space of the anterior neck. The NGT was removed and surgical debridement was performed to evacuate the milk contamination. The case illustrates the importance of radiological confirmation of NGT placement in the ventilated patient. 